\title{
A Novel APPROACH FOR HANDOFF AVOIDANCE AND QOS IMPROVEMENT IN WLAN
}

\author{
V. Berlin Hency ${ }^{1}$, S. Aravind Prasad ${ }^{2}$, Y.RA. Kannan ${ }^{2}$ and D. Sridharan ${ }^{3}$ \\ ${ }^{1}$ Visiting Faculty, Department of Information Technology, Madras Institute of \\ Technology, Chennai-600044. \\ hencyjosepheannauniv .edu \\ ${ }^{2}$ Department of Information Technology, Madras Institute of Technology, \\ Chennai-600044. \\ raja.avi@gmail.com, yra.kannan@gmail.com \\ ${ }^{3}$ Department of ECE, College of Engineering, Guindy, \\ Chennai- 600025 \\ Sridhareannauniv .edu
}

\begin{abstract}
Maximizing network throughput while providing fairness is one of the key challenges in wireless LANs (WLANs). Dense deployments of WLANs suffer from increased interference and, as a result, reduced capacity and wireless network conditions in general are diverse owing to both traffic and wireless channel variations. Hence effective mechanisms are required to exploit the diversity across a multiple access points and to provide a uniform load distribution in order to improve the QoS of users and to improve the overall composite capacity. In this paper, we propose a handoff scheme considering the current active application in use rather than a single fixed threshold value and dynamically changing the threshold value depending upon the currently active applications. In addition to it, we also propose a pro-active Load Balancing scheme which completely avoids unnecessary overloading of access points by considering the load at the target access point before associating with it.
\end{abstract}

\section{KEYWORDS}

WLANs, interference, Handoff, Fixed threshold, QoS, Load Balancing, Active applications.

\section{INTRODUCTION}

Wireless Local Area Network (WLAN) systems, which was basically designed to eliminate the problem caused due to tangled wired cables among network devices in indoor environments has developed to a great extent in supplying high data rates along with providing seamless mobility and extensible support to multimedia communications. Further, the ability to support seamless services greatly depends upon the extent to which IEEE 802.11 systems can support fast handoffs in their infrastructures. In addition to it, the average handoff delay is required to be much lesser than the current delay restrictions.

Usually, expected Ideal scenario in a wireless network is maximum utilization of system resources while maintaining a good Quality of service to its users. But practically, with realtime VoIP and video services over WLAN becoming popular nowadays, a great amount of work is needed in the areas of QoS maintenance and admission control within the network. A Typical scenario may include considerable number of hotspots deployed in a distributed fashion throughout the network in order to cope up with the increasing demands of traffic flow in the network.

But this scenario becomes more and more complicated if the selection of AP's by the stations are purely based on received signal without considering the QoS parameters such as load 
International Journal of Distributed and Parallel Systems (IJDPS) Vol.2, No.5, September 2011

control and control over the provisional distribution. And in the cases where load distribution of APs are not uniform, it further adds to complexity of problem since there is a high probability of users correlating with each other both spatially and temporally finally leading to sporadic congestions in the network. Hence, considerable effort is needed to exploit the diversity and complete capacity including the QoS improvements within the network.

Generally, Handoff is initiated as soon as MH changes its association from one AP to another. Usually, this situation is identified by the decrease in Received Signal Strength (RSS), which may go below the required threshold level. Hence, the most important issue is to discover the next best AP, which could support the station with acceptable RSS value. Hence usually, the $\mathrm{MH}$ tries to to actively probe the channels for the best available AP (discovery phase) and associate with the selected AP (reauthentication phase).Here, the channel scanning time plays a crucial factor in supporting applications since it predominantly occupies most of handoff delay period. Also, in case of delay sensitive applications like Voice over IP (VoIP) and Multimedia applications, QoS maintenance has to be guaranteed.

Considering the importance of channel scanning phase, there has been some mechanisms followed to overcome it as far as possible. (i) selective scanning approach, which selects some deterministic channels to develop a channel mask out of all probable ones. Hence, as long as $\mathrm{MH}$ discovers the next best available AP with channel mask, handoff delay could be considerably avoided. But, in the worst case, the entire channel scanning is repeated incase of not discovering the next AP to be associated with. (ii) First Satisfaction First Reservation (FSFR), which opts for the first suitable AP to be associated with rather than choosing an AP with highest RSS value. The greatest advantage here is that, we do not perform the full channel scanning as we are satisfied as soon as we find a suitable AP with sufficient (not highest) RSS value. But the disadvantage is that, we may enter into situations where further handoffs may be required depending upon the proximity of location of network stations.

Traditionally, load control mainly deal with distribution of load in the network by transfer of stations from heavily to lightly loaded networks. This helps in exploiting the spare network capacity which may otherwise be left unused. Moreover, it is almost crucial to review the properties and state of wireless channel, which could pose a fundamental roadblock on the performance and QoS in network, when distributing load across wireless networks. Usually, admission control policy provides the overview of either completely guaranteed or predictive QoS. Hence, a complete knowledge of load metric is certainly required while designing the load and admission control in considered network.

Now all the above mechanisms make use of single fixed threshold value without considering the current active application in use. And the load balancing algorithms previously proposed by various authors are generally reactive in nature and hence implemented only after the AP gets overloaded. In this paper, we propose a handoff scheme considering the current active applications in use rather than a single fixed threshold value and dynamically changing the threshold value depending upon the currently active applications. In addition to it, we also propose a pro-active Load Balancing scheme which completely avoids unnecessary overloading of access points by considering the load at the target access point before associating with it.

The remainder of the paper is organized as follows. Section 2 describes our proposed mechanism. Section 3 illustrates the comparative performance evaluation. Section 4 discusses the related work and Section 5 represents the conclusion.

\section{Proposed Mechanism}

We propose two influencing factors: 1) Application Specific Threshold (AST) and 2) Load Balancing at each Access point (AP). 


\subsection{Application Specific Threshold}

Usually when the Mobile Host (MH) moves away from the currently associated AP, RSS value from that AP gets reduced below the fixed threshold value and then handoff occurs to another AP by means of active scanning process. Threshold value for handoff process is fixed irrespective of the currently active applications in MH. For e.g. the currently active application in MH requires only less amount of RSS value, say $-85 \mathrm{~dB}$ and if the threshold value is fixed to $-60 \mathrm{~dB}$, then the unnecessary handoff would occur even though the $\mathrm{MH}$ is receiving required RSS when it moves away from the currently associated AP. Hence, according to our proposed mechanism each MH should maintain AST i.e. the threshold value with respect to the current active application in use rather than a single fixed threshold value. If the number of applications that are currently in use is more than one then the maximum AST should be selected among AST corresponding to the respective applications. For e.g. if there are three active applications in MH, say AST of Application I is $-60 \mathrm{~dB}$, AST of Application II is $-90 \mathrm{~dB}$ and AST of Application III is $-50 \mathrm{~dB}$ then AST corresponding to Application III is selected since it is maximum among the three applications. The AST selection is done in the application layer of $\mathrm{MH}$ and this selection process should be dynamic in such a way that maximum AST should be selected if there are changes in the number of applications that are currently in use. There should be cross layer communication between Application Layer and MAC layer to deliver the information about AST. The main advantage of AST is when the MH is at the edge of an AP's network coverage area, then RSS from the current AP will be low and if this RSS is greater than required AST then unnecessary handoff can be avoided.

\subsection{Load Balancing}

Various load metrics can be considered for Load Balancing such as the number of MHs that can be connected to an AP, the bandwidth received by the user from an AP. In our proposed mechanism, we are considering the load metric as number of MHs connected to AP. Load Capacity Threshold (LCT) indicates the maximum number of MHs that can be connected with an AP and Load Capacity of AP (LCAP) indicates the number of MHs that are currently associated with an AP i.e. the number of active connections. Initially, all APs should broadcast its LCAP to its neighboring AP. Hence each AP knows Load Capacity of its neighboring APs. Each AP has to maintain a list which consists of Load Capacity of neighboring APs and the values of LCAP maintained in this list should be sorted in ascending order. Hence, the first LCAP value in the list indicates that AP is under loaded and the last LCAP value in the list indicates that AP is overloaded. If two LCAP values are same then the values are stored as per FCFS procedure. The list maintained by each AP should be dynamic i.e. if there are any changes in the number of MHs that are associated with an AP then the corresponding AP should broadcast its LCAP to its neighboring APs. Hence, each AP maintains an updated list of LCAPs.

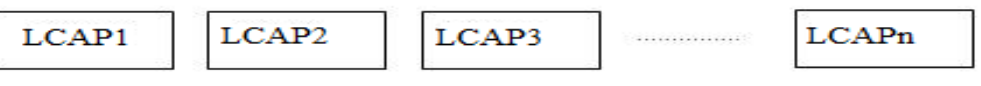

Figure 1. List Maintained by each AP 


\subsection{Overall Process}

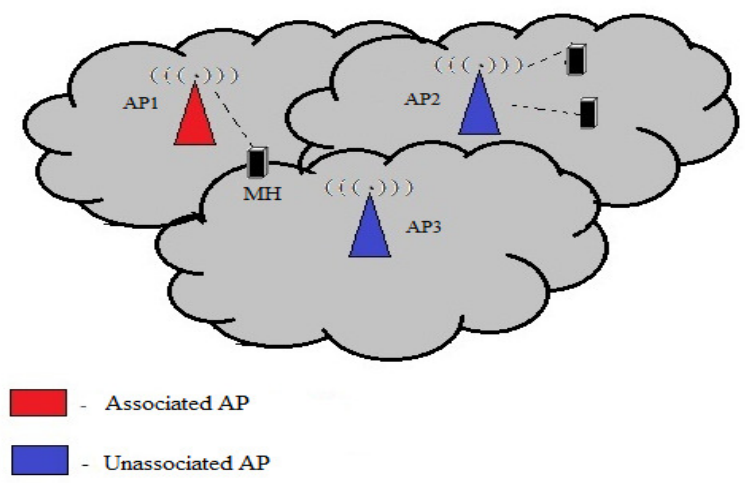

Figure 2. MH is associated with AP1

Consider MH is currently associated with AP1 and it moves far away from AP1.

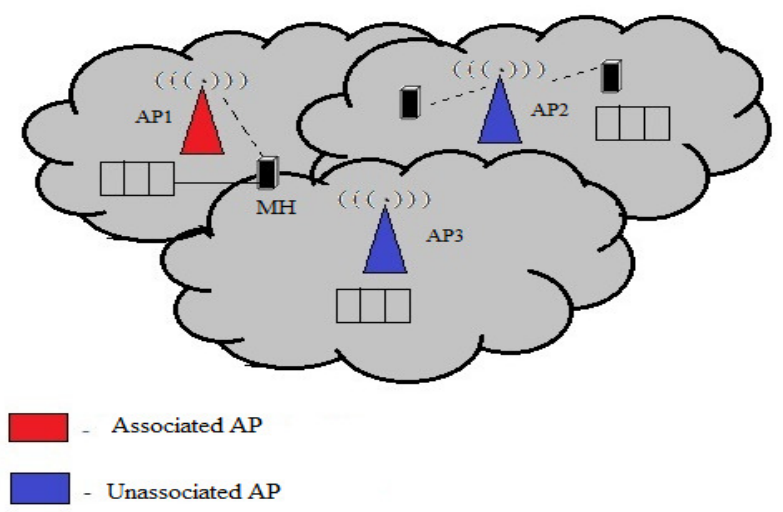

Figure 3. RSS $<$ AST, MH accesses the list maintained by AP1

Consider the situation that RSS received from AP1 goes below the required AST value. At this time, Handoff will be initiated. Now the MH accesses the list maintained by AP to which it is currently associated as shown in Figure 3.

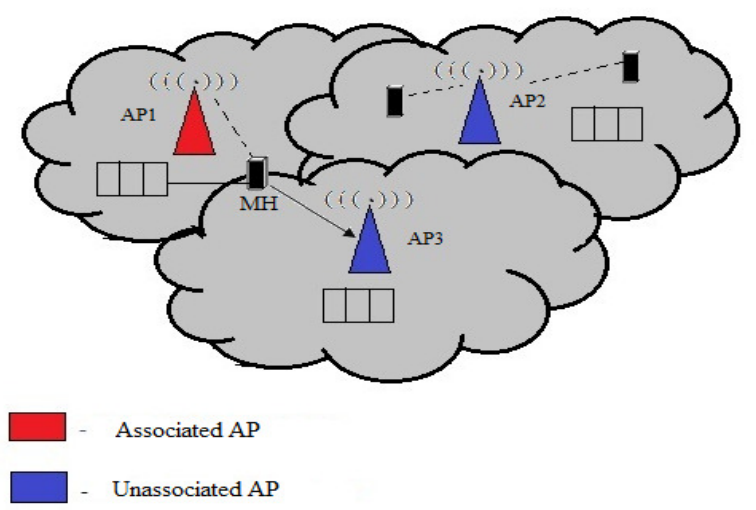


Figure 4. MH chooses AP with acceptable RSS value > AST value based on FSFR-HO Mechanism

Since the list is maintained in the increasing order of Load Capacity of neighboring APs, MH accesses the list and compares the RSS value received from each AP in the list with its AST. This process is continued as per FSFR-HO mechanism until an AP with an acceptable RSS value is reached. MH selects AP3 by accessing the list since RSS received from AP3 is greater than AST and also AP3 is not overloaded. MH chooses AP3 by accessing the list that is maintained by AP1. MH checks the RSS value received from AP3 with AST. If RSS value is acceptable then it gets associated with AP3.

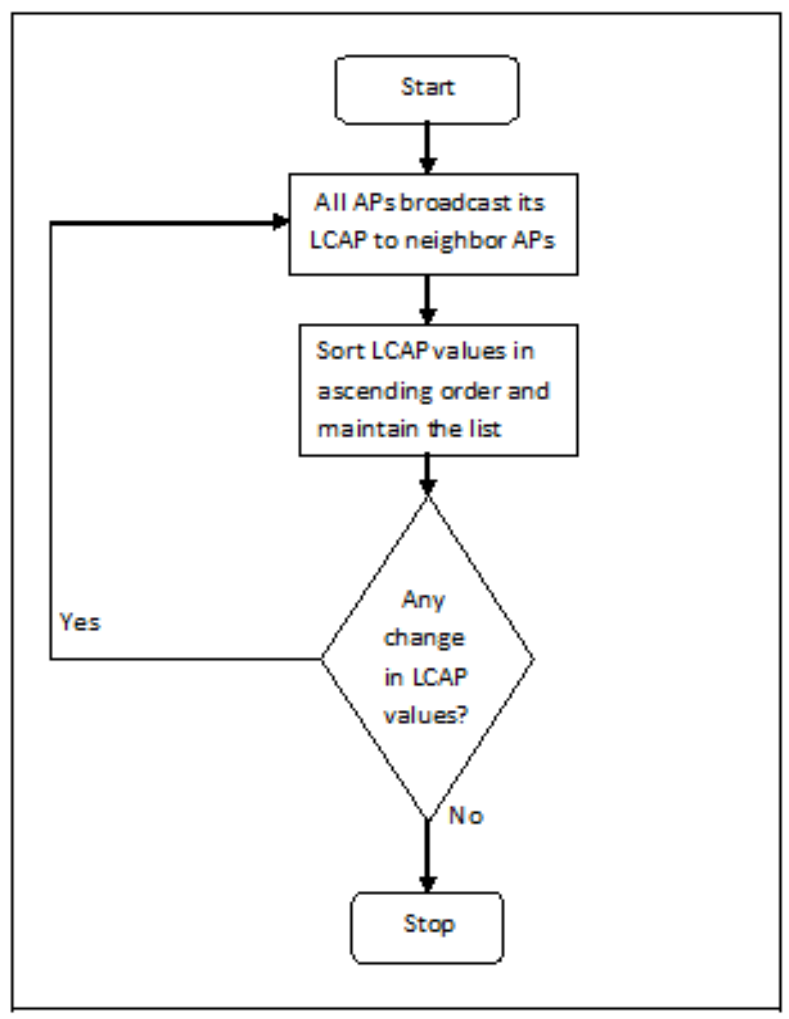

Figure 5. List Maintenance Procedure at each AP

Figure 5 shows the List Maintenance Procedure at each AP. This is the initial procedure that should be implemented by each AP and the list should be broadcasted to its neighbor APs. If there are any changes in LCAP value of an AP then that AP should broadcast its LCAP value to its neighboring APs. Therefore the updated list should be maintained by each AP dynamically.

The following Figure 6 shows the steps in Cognitive Handoff Procedure. Normal Operation in the following figure indicates the normal data transfer operation between $\mathrm{MH}$ and AP without any handoff procedure. If RSS from the associated AP falls below AST of MH then the Cognitive Handoff Procedure begins as given in the Figure 6. After selecting the best target AP according to Cognitive Handoff Procedure, MH will be associated with the selected AP. 
International Journal of Distributed and Parallel Systems (IJDPS) Vol.2, No.5, September 2011

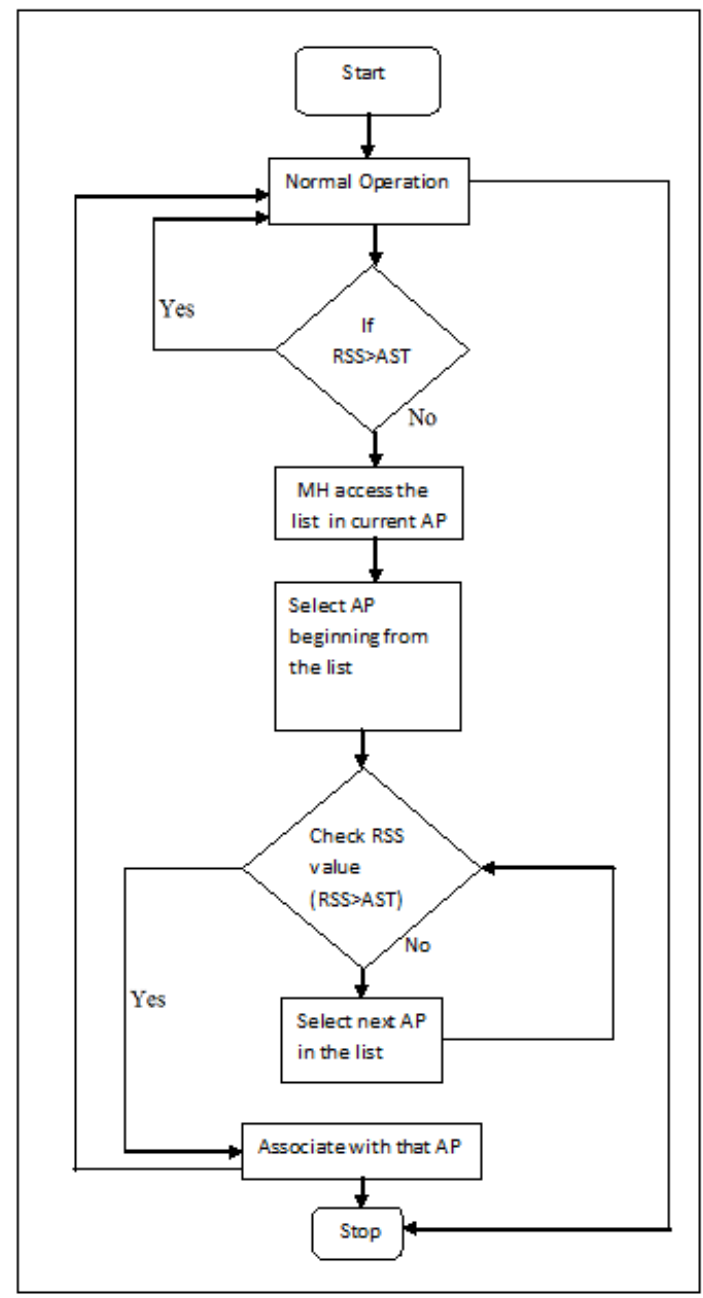

Figure 6. Cognitive Handoff Procedure

Hence, our proposed mechanism provides three main benefits, viz. (i) avoiding unnecessary handoffs, since the Application Specific Threshold is considered (ii) improving QoS of all MHs connected to each AP since AP with minimum load capacity is considered first (iii) MH will be connected to the best target AP during handoff (AP with minimum load and providing satisfiable RSS).

\section{Performance Evaluation}

In this section, we show performance evaluation of the proposed Cognitive Handoff Mechanism (CHM) mechanism compared to the Traditional Handoff Mechanism (THM). Figure 7 and 8 present the average total handoff delay time and the Average Throughput achieved by CHM and THM respectively. 


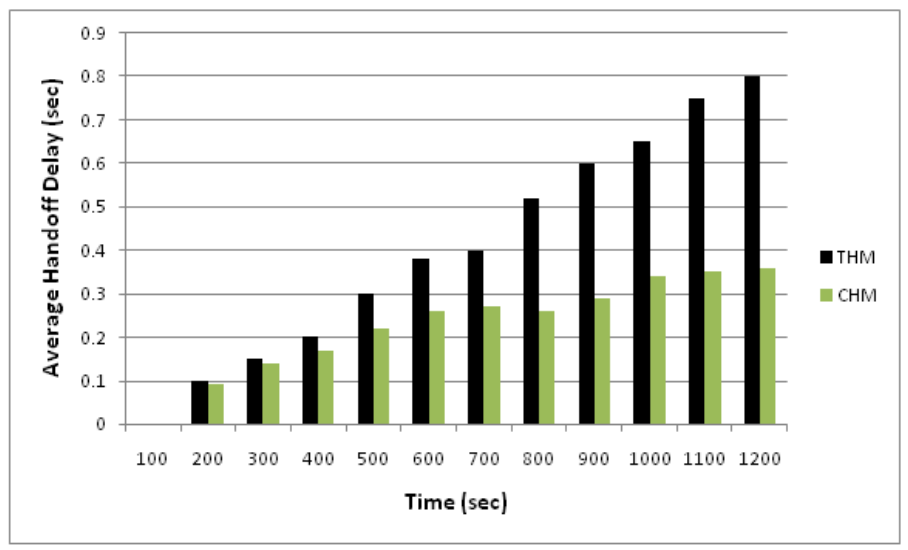

Figure 7. Average Handoff Delay Vs Time

For our simulations we set the fixed threshold value as $-15 \mathrm{~dB}$ and made use of 3 different applications APP1, APP2 and APP3 with 3 different threshold values of $-5 \mathrm{~dB},-8 \mathrm{~dB}$, and -13 $\mathrm{dB}$ respectively. The Load Capacity Threshold (LCT) for each AP as fixed as 5 and as a whole, 5 APs was used for our simulation purposes. Across our simulations we increased the number of MHs from 3 to upper bound of $20 \mathrm{MHs}$ to stress on the improvement in system performance for large loads. In THM mechanism, since the handoff occurs depending upon fixed threshold value $(-15 \mathrm{~dB})$ irrespective of the active application in use, in the extreme situations (i.e. when the MH reaches the edge of the current AP range) handoff would have occurred even though the current active application in $\mathrm{MH}$ is able to receive satisfiable RSS. Hence the overall delay time increases due to handoff initiation and scanning for all probable channels. Under similar conditions, the performance of CHM improves greatly since the RSS that the MH receives from the current AP satisfies with AST which, in most situations, is less than the predefined value. Hence with the implementation of CHM mechanism, handoffs could be cognitively avoided which greatly aids in decreasing the overall delay while improving the network throughput and performance.

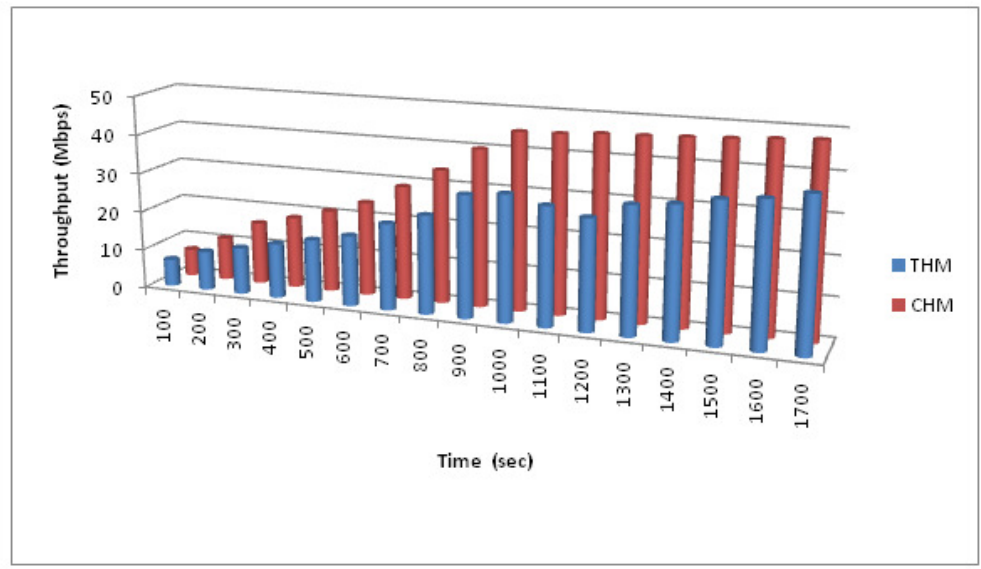

Figure 8. Throughput Vs Time 
International Journal of Distributed and Parallel Systems (IJDPS) Vol.2, No.5, September 2011

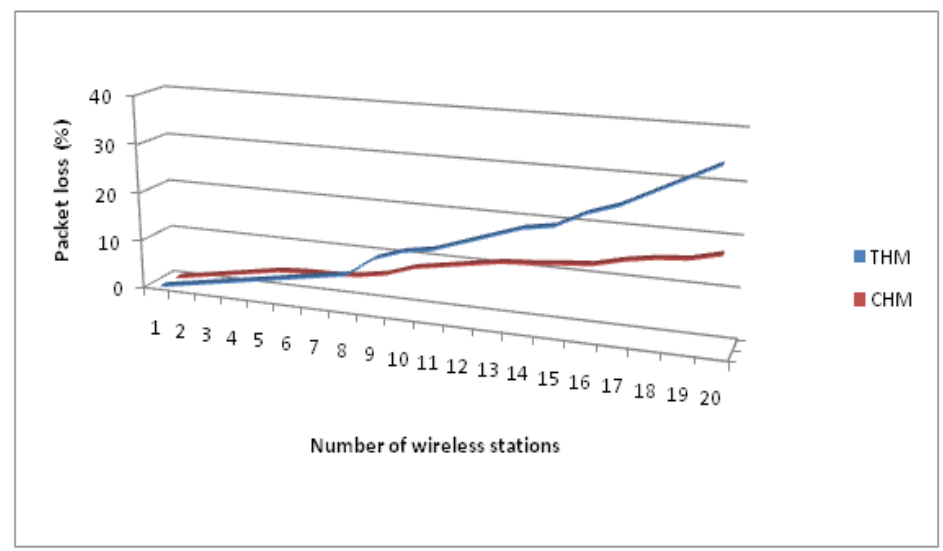

Figure 9. Packet loss Vs Number of wireless stations

In case of Load balancing too, our proposed pro-active cognitive Load Balancing mechanism (CHM) outperforms the traditionally mechanism (THM) since the load at the target access point is considered before associating with it. The simulation results clearly show that, in case of large loads the performance and overall throughput of THM degrades compared to our proposed scenario. And there is also a significant decrease in the amount of packet losses in the network in our proposed scenario. Additionally in case of traditional mechanisms, there is a possibility of already overloaded AP to be associated with further more MHs (more than its LCT) and which may lead to decrease in QoS of not only the new MH but also for the already associated MHs. Whereas in our proposed mechanism, this possibility is essentially eliminated as the MH has the advantage of selecting the AP in its vicinity with minimum load and receive an acceptable RSS value (RSS >AST).

\section{RELATED WORK}

Several studies proposed different load balancing schemes with different load balancing metrics. Li-Hsing Yen et al. [6] proposed that balancing AP traffic loads effectively can increase overall system performance. The metrics that had been taken into account for load balancing in several schemes are the number of users associated with an AP, the bandwidth that a new user can get from an AP if that user is connected with that AP. In [2], Yigal Bejerano et al. proposed Cell Breathing Technique for Load Balancing in wireless LAN by controlling the size of WLAN cells. For changing the size of cells, the ability to dynamically change the transmission power of AP beacon messages is required. In this approach, when the AP becomes overloaded then handoff occurs by reducing the cell size. Hence it leads to more handoff occurrence for the mobile nodes.

Ahmed H. Zahran et al. [10] proposed the effect of Application Signal Strength Threshold values depending on the packet delay, bandwidth and number of handoffs. If the Application Signal Strength Threshold value is reduced, the mobile node can be connected with the associated AP for longer duration. In [4], Yang Song et al. proposed that threshold based rate adaptation algorithms play a vital role in IEEE 802.11 services. The System performance can be increased if the threshold is tuned optimally. Eng Hwee Ong et al. [11] proposed the integrated load balancing scheme which includes the QoS based Handover and soft admission control to provide seamless connectivity. But this approach requires the additional Access point controller which gathers the information from each access points and also leads to single point of failure. Yazan M. Allawi et al. [12] proposed First Satisfaction First Reservation Handoff (FSFR-HO) Mechanism i.e. while discovering the next access point during handoff, the access point which has satisfactory Received signal strength value is selected rather than scanning all the access points and choosing the best one. Eng Hwee Ong et al. [1] provided a comparison 
International Journal of Distributed and Parallel Systems (IJDPS) Vol.2, No.5, September 2011

between three dynamic load distribution algorithms, viz. predictive load balancing (PLB), predictive $\mathrm{QoS}$ balancing $(\mathrm{PQB})$ and reactive $\mathrm{QoS}$ balancing (RQB) based on opnet simulations QoS metrics considered were packet delay and packet loss rate. It was shown that RQB achieves higher QoS fairness compared to PQB at admission thresholds of $80 \%$ to $90 \%$. Our proposal differs from the above mentioned works in the following ways (i) Handoff could be avoided by making use of Application Specific Threshold value, (ii) Improving QoS for each MH by using distributed Load Balancing scheme at each access point based on the load capacity and the application specific threshold value.

\section{CONCLuSiON}

In this paper, we propose a handoff scheme considering the current active application in use rather than a single fixed threshold value and dynamically changing the threshold value depending upon the currently active applications which greatly helps incase of delay sensitive applications since handoffs can be cognitively avoided in extreme situations thereby avoiding the unnecessary scanning of channels and delays associated with it. In addition to it, we also propose a pro-active Load Balancing scheme which completely avoids unnecessary overloading of access points by considering the load at the target access point before associating with it. Hence, our proposed mechanism provides three main benefits, viz. (i) avoiding unnecessary handoffs (since the Application Specific Threshold is considered) (ii) improving QoS of all MHs connected to each AP (since AP with minimum load capacity is considered first) (iii) $\mathrm{MH}$ will be connected to the best target AP during handoff (AP with minimum load and providing satisfiable RSS).

\section{REFERENCES}

[1] E.H. Ong, J.Y. Khan, K. Mahata, (2010) "On Dynamic Load Distribution Algorithms for Multi-AP WLAN under Diverse Conditions,” Proceedings of WCNC 2010, IEEE Press.

[2] Y. Bejerano, S. Han, (2009) "Cell Breathing Techniques for Load Balancing in Wireless LANs," IEEE Transactions on Mobile Computing, IEEE Press.

[3] I. Broustis, K. Papagiannaki, S.V. Krishnamurthy, M. Faloutsos, V.P. Mhatre, (2010) "MeasurementDriven Guidelines for 802.11 WLAN Design," IEEE/ACM Transactions on Networking, IEEE Press.

[4] Y. Song, X. Zhu, Y. Fang, H. Zhang, (2010) “Threshold Optimization for Rate Adaptation Algorithms in IEEE 802.11 WLANs,” IEEE Transactions on Wireless Communications, IEEE Press.

[5] S. Shin, H. Schulzrinne, (2009) "Measurement and Analysis of the VoIP Capacity in IEEE 802.11 WLAN," IEEE Transactions on Mobile Computing, IEEE Press.

[6] L.H. Yen, T. Yeh, K.H. Chi, (2009) "Load Balancing in IEEE 802.11 Networks," IEEE Computer Society, IEEE Press.

[7] M. Bshara, U. Orguner, F. Gustafsson, L.V. Biesen, (2010) "Fingerprinting Localization in Wireless Networks Based on Received-Signal-Strength Measurements," IEEE Transactions on Vehicular Technology, IEEE Press.

[8] S.D. Chitte, S. Dasgupta, Z. Ding, (2009) "Distance Estimation From Received Signal Strength Under Log-Normal Shadowing: Bias and Variance,” IEEE Signal Processing Letters, IEEE Press.

[9] C.T. Huang, C.H. Wu, Y.N. Lee, J.T. Chen, (2009) "A Novel Indoor RSS-Based Position Location Algorithm Using Factor Graphs," IEEE Transactions on Wireless Communications, IEEE Press.

[10] A.H. Zahran, B. Liang, A. Saleh, (2005) "Signal Threshold Adaptation for Vertical Handoff in Heterogeneous Wireless Networks," ACM/Springer Mobile Networks and Applications (MONET), Springer.

[11] E.H. Ong, J.Y. Khan, (2009) “An Integrated Load Balancing Scheme for Future Wireless Networks," IEEE Press. 
International Journal of Distributed and Parallel Systems (IJDPS) Vol.2, No.5, September 2011

[12] Y.M. Allawi, M.G. Kim, M. Kang, (2008) “Advanced Handoff Mechanism for Delay Sensitive Applications in IEEE 802.11 Wireless LAN,” ICACT 2008, IEEE Press.

[13] Y.M. Allawi, M.G. Kim, M. Kang, (2008) "Enhanced Scanning Scheme for Delay-Constrained Fast Handoff in IEEE 802.11 WLANs,” IEEE Press.

\section{Authors}

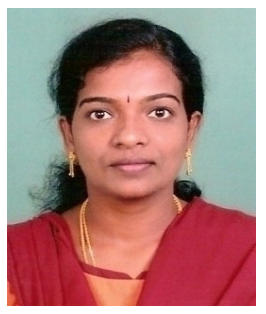

V. Berlin Hency Received her BE degree in Electronics and communication Engineering from Manonmaniam Sundaranar University India, ME degree in Applied Electronics from Sathyabama University India, Pursuing her $\mathrm{PhD}$ Degree in Information and Communication Engineering from Anna University, India. She is working as a lecturer in MIT campus, Anna University, Chennai, India. Her research interests include wireless multimedia networks, mobility management, mobile computing, and mobile IP. She is a member of IACSIT and the life member of Indian Society for Technical Education(ISTE).

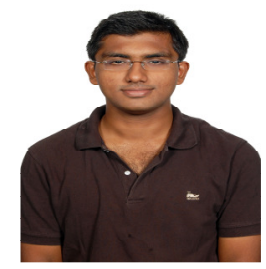

S. Aravind Prasad received the B.Tech degree in Information Technology from the Madras Institute of Technology, Anna University, Chennai, India in 2011. $\mathrm{He}$ is currently working as Chief Technology Officer in Dell and is an active member of IEEE Computer society. His research interests include mobile computing, mobility management, scheduling, resource management and quality of service issues in wireless and high speed networks.

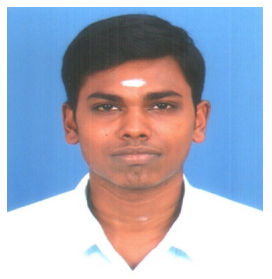

Y.RA.Kannan received his B.Tech degree in Information Technology from Madras Institute of Technology, Anna University, India in 2011. He is currently working as Network Engineer in Dell. His research interests include switching and routing technology in wired networking, wireless multimedia networks, Cryptography, mobile computing and mobile IP. He is an active member of IEEE computer societv.

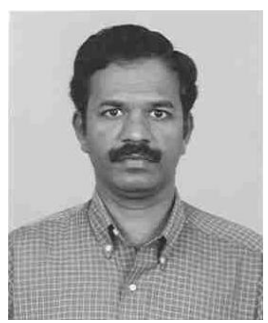

Dr. D. Sridharan received his B. Tech degree in Electronics Engineering and M.E. degree in Electronics Engineering from Madras Institute of Technology, Anna University in the years 1991 and 1993 respectively. He got his $\mathrm{Ph}$. D. degree in the Faculty of Information and Communication Engineering, Anna University in 2005. $\mathrm{He}$ is currently working as Associate Professor in the Department of Electronics and Communication Engineering, CEG Campus, Anna University, Chennai, India. He was awarded the Young Scientist Research Fellowship by SERC of Department of Science and Technology, Government of India. His present research interests include Internet Technology, Network Security, Distributed Computing and VLSI for wireless Communications. He has published more than 25 papers in National/International Conferences and Journals. He is a life member of Institution of Electronics and Telecommunication Engineers (IETE), Indian Society for Technical Education(ISTE)and Computer Society of India(CSI). Presently he is working on a research project on Wireless Sensor Network sponsored by Department of Atomic Energy. 